\title{
Carboxyamidotriazole Synergizes with Sorafenib to Combat Non-Small Cell Lung Cancer through Inhibition of NANOG and Aggravation of Apoptosis
}

\author{
Chen Chen, Rui Ju, Jing Shi, Wei Chen, Fangrui Sun, Lei Zhu, Juan Li, Dechang Zhang, \\ Caiying Ye, and Lei Guo \\ Department of Pharmacology, Institute of Basic Medical Sciences, Chinese Academy of Medical Sciences and School of Basic \\ Medicine, Peking Union Medical College, Beijing, China
}

Received February 25, 2017; accepted May 16, 2017

\begin{abstract}
Lung cancer is currently the leading cause of cancer-related deaths worldwide. In this study, we investigated the combination of carboxyamidotriazole (CAl) and sorafenib in non-small cell lung cancer (NSCLC) in vitro and in vivo to test whether CAl enhances the antitumor effects of sorafenib and reduces its side effects. The combination index $(\mathrm{Cl})$ showed that coadministration of CAl and sorafenib synergistically inhibited the proliferation of NSCLC cells (Lewis lung carcinoma, A549, and NCl-H1975 cells). Cell death as a result of the combination treatment was attributed to apoptosis, which was accompanied by activation of caspase-3 and poly(ADP-ribose) polymerase. In addition, combination therapy induced the accumulation of mitochondrialassociated reactive oxygen species, as well as depolarization of mitochondrial and reduced NANOG (homeobox protein NANOG) mRNA and protein expression. Basic fibroblast growth factor, a stimulator of NANOG, was applied to identify the possible
\end{abstract}

mechanism. The addition of basic fibroblast growth factor followed by combined treatment may stimulate NANOG expression and synchronously rescue the accumulation of reactive oxygen species. C57BL/6J mice bearing Lewis lung carcinoma were randomized to receive vehicle (polyethylene glycol 400), CAl (30 mg/kg), low-dose sorafenib (SFB-L; $10 \mathrm{mg} / \mathrm{kg}$ ), high-dose sorafenib (SFB-H; $30 \mathrm{mg} / \mathrm{kg}$ ), or a CAI and SFB-L combination. Tumor growth was significantly suppressed in the combination group, and the efficacy of combination treatment was equivalent to that of the SFB-H monotherapy group. Furthermore, the combination group had reduced side effects compared with the SFB-H group, as indicated by weight preservation in mice. Our study illustrates that CAl enhances the antitumor activity of sorafenib in NSCLC and provides a novel strategy for NSCLC treatment.

\section{Introduction}

Lung cancer is currently the leading cause of cancer-related death worldwide, and the 5-year survival of patients with lung cancer is approximately $4 \%-17 \%$, with variations in disease stage and region (Hirsch et al., 2017). Non-small cell lung cancer (NSCLC) accounts for $80 \%$ of all lung cancers worldwide (Jemal et al., 2011). The most frequently used treatments for NSCLC are surgery, chemotherapy, and radiotherapy. Unfortunately, approximately two-thirds of patients have advanced or inoperable disease at diagnosis. Many platinum-based regimens are firstline chemotherapies; single-agent docetaxel, pemetrexed, and

This research was supported by the Ministry of Science and Technology of China [Major Scientific and Technological Special Project 2014ZX09507003003], the National Science Foundation of China [Grant 81402943], the Chinese Academy of Medical Sciences Major Collaborative Innovation Project [Grant 2016-I2M-1-011], and the Peking Union Medical College Youth Fund [Grant 3332015168].

https://doi.org/10.1124/jpet.117.240986. erlotinib are considered second-line therapies. However, if patients fail to respond to conventional chemotherapies, treatment options are limited (Langer et al., 2013). The currently available therapies are shown to be inadequate, and novel strategies are urgently required.

Sorafenib is a multikinase inhibitor that blocks the Raf kinase, platelet-derived growth factor receptors, and vascular endothelial growth factor receptors (EGFRs). The U.S. Food and Drug Administration approved sorafenib for the treatment of advanced renal cell carcinoma and unresectable hepatocellular carcinoma (Wilhelm et al., 2006). Sorafenib has confirmed activity in preclinical models of NSCLC (Wilhelm et al., 2004; Gridelli et al., 2007) and has also been evaluated in several clinical trials (phases I-III) in patients with advanced NSCLC (Clark et al., 2005; Moore et al., 2005; Blumenschein et al., 2009; Wakelee et al., 2012; Paz-Ares et al., 2015). One limitation that hampers the use of sorafenib as a monotherapy is its side effects (e.g., hand-foot syndrome, rash, diarrhea, hypertension, and fatigue), which affect approximately $80 \%$ of patients receiving

ABBREVIATIONS: bFGF, basic fibroblast growth factor; CAI, carboxyamidotriazole; Cl, combination index; CSC, cancer stem cell; DCFH-DA, $2^{\prime}, 7^{\prime}$-dichlorofluorescein diacetate; DMSO, dimethylsulfoxide; EGFR, endothelial growth factor receptor; FCM, flow cytometry; GSH, glutathione; LLC, Lewis lung carcinoma; MDA, malondialdehyde; NSCLC, non-small cell lung cancer; OXFOS, oxidative phosphorylation; PARP, poly(ADPribose) polymerase; PBS, phosphate-buffered saline; PEG400, polyethylene glycol 400; PI, propidium iodide; ROS, reactive oxygen species; SFB-H, high-dose sorafenib; SFB-L, low-dose sorafenib; SRB, sulforhodamine B; Z-VAD-FMK, Benzyloxycarbonyl-Val-Ala-Asp (OMe) fluoromethylketone. 
sorafenib treatment (Batchelor et al., 2007; Strumberg et al., 2007). Most of the aforementioned side effects are dose dependent; therefore, reducing the sorafenib dose while maintaining the same therapeutic effect may require further investigation.

Carboxyamidotriazole (CAI) blocks nonvoltage-dependent calcium channels and has shown antiangiogenic, antiproliferative, and antimetastatic properties in preclinical research. CAI has been investigated in phase I-III clinical trials in various solid tumors (Berlin et al., 1997; Kohn et al., 1997; Hussain et al., 2003; Dutcher et al., 2005; Johnson et al., 2008). Although CAI exhibited mild anticancer properties in some clinical trials, the majority of toxicities noted were grade I, which means CAI was generally well tolerated (Hussain et al., 2003; Desai et al., 2004; Dutcher et al., 2005; Mikkelsen et al., 2007). In addition, CAI exhibited a protective role in treating cancer-associated cachexia by inhibiting muscle proteolysis and restraining inflammatory responses (Chen et al., 2017), which implies that CAI may synergize with other anticancer drugs through limiting chemotherapy-induced weight loss. In chronic myeloid leukemia cells, CAI was shown to reduce cell viability and induce apoptosis in a redox-mediated way (Alessandro et al., 2008; Corrado et al., 2011). Our previous work showed that CAI synergized with 2-deoxy-D-glucose, a glycolysis inhibitor, and inhibited oxidative phosphorylation (OXPHOS) in cancer cells (Ju et al., 2016). These data imply that CAI may perturb cell metabolism, damage mitochondrial function, elevate reactive oxygen species (ROS) production, and, finally, induce cell death.

The NANOG transcription factor confers self-renewal and differentiation to cancer stem cells (CSCs) and is often considered a hallmark of CSCs. Recently accumulated data show that the expression of NANOG in cancer cells can make confer them certain CSC properties such as self-renewal, tumorigenicity, metastasis, and chemotherapy resistance (Gong et al., 2015). Overexpression of NANOG in benign 293 cells contributed to malignant transformation, along with tumorigenesis, dramatically increasing clonogenicity and promoting tumor growth in vivo (Lin et al., 2011). In patients with lung cancer, expression of both NANOG mRNA and protein in lung cancer tissues was much higher than in patient-matched normal tissues (Du et al., 2013). Regarding the signaling perturbed by NANOG within cells, Chen et al. (2016) showed that NANOG prevented mitochondrial ROS production in tumorinitiating stem-like cells (TICs), and NANOG-silenced TICs produced much more ROS than controls (Chen et al., 2016).

Our objectives were first to probe whether combination treatment with CAI and sorafenib may allow a dose reduction of sorafenib and avoid decreased effectiveness and then to investigate the mechanism by which the combination exerts its action.

\section{Materials and Methods}

Cell Lines and Reagents. Lewis lung carcinoma (LLC) cells were purchased from the Shanghai Institute for Biologic Sciences (Chemical Abstracts Service no. A549; Shanghai, China) and NCI-H1975 (H1975) cells were purchased from the Institute of Basic Medical Sciences at the Chinese Academy of Medical Sciences and School of Basic Medicine, Peking Union Medical College (Beijing, China). LLC cells were cultured in Dulbecco's modified Eagle's medium (high glucose). A549 cells were maintained in F-12K medium. H1975 was grown in RPMI 1640 medium. All media were supplemented with $10 \%$ fetal bovine serum (Gibco, Grand Island, NY), $50 \mathrm{mg} / \mathrm{ml}$ penicillin, and $100 \mathrm{mg} / \mathrm{ml}$ streptomycin and L-glutamine. Cells were maintained in a humidified atmosphere with $5 \% \mathrm{CO}_{2}$ at $37^{\circ} \mathrm{C}$.

CAI was synthesized by the Institute of Materia Medica at the Chinese Academy of Medical Sciences (Beijing, China). Sorafenib was purchased from MedChem Express (Monmouth Junction, NJ). Glutathione (GSH)-reduced ethyl ester (Sigma-Aldrich, St. Louis, MO) was dissolved in sterile phosphate-buffered saline (PBS) as a $0.25-\mathrm{M}$ stock solution. Benzyloxycarbonyl-Val-Ala-Asp (OMe) fluoromethylketone (Z-VAD-FMK) (Beyotime Biotechnology, Nantong, China) was prepared in dimethylsulfoxide (DMSO) as a $20-\mathrm{mM}$ stock solution. For in vitro studies, CAI and sorafenib were dissolved in DMSO and diluted with the corresponding medium to the final concentration with a DMSO concentration of $0.1 \%$. For in vivo studies, compounds were dissolved in polyethylene glycol 400 (PEG400) (Sinopharm Chemical Reagent, Beijing, China).

Cell Proliferation Assay and Combination Index. The sulforhodamine B (SRB) method was used to assess cell proliferations for different concentrations after the indicated treatment times (Vichai and Kirtikara, 2006). DMSO was used as the vehicle. After treatment with CAI and/or sorafenib for the indicated time period ( 24 hours, 48 hours, and 72 hours), cells were fixed in 96 -well plates with $10 \%(\mathrm{w} / \mathrm{v})$ trichloroacetic acid at $4^{\circ} \mathrm{C}$ for 1 hour, and the plates were then washed with slow-running water five times and dried at room temperature. Next, the intracellular proteins were stained with $0.4 \%$ SRB for 20 minutes, and the plates were then washed five times with $1 \%(\mathrm{v} / \mathrm{v})$ acetic acid. After adding $200 \mu \mathrm{l}$ of $10 \mathrm{mM}$ Tris base solution to each well, the absorbance of SRB was detected at $515 \mathrm{~nm}$ in a microplate reader. Combination indices (CIs) were calculated using CompuSyn software (ComboSyn Inc., Paramus, NJ) (Chou and Talalay, 1984; Chou, 2006). According to Chou and Talalay (1984), a CI less than 1 or greater than $>1$ indicates synergism or antagonism, respectively.

Apoptosis and Cell Cycle Analysis by Flow Cytometry. Cells were seeded and treated with CAI and/or sorafenib for 24 hours, and they were then detached from the plates with Trypsin-EDTA and washed with PBS two times. The induction of apoptosis was measured by flow cytometry (FCM) using an Annexin V-fluorescein isothiocyanate/propidium iodide (PI) kit (Dojindo, Kyushu, Japan). The approximate fluorescence excitation/emission wavelengths of fluorescein isothiocyanate-Annexin $\mathrm{V}$ and propidium iodide were $494 / 518 \mathrm{~nm}$ and $488 / 617 \mathrm{~nm}$, respectively. Both the Annexin V-and PI-negative subpopulation indicated intact cells, whereas the Annexin V-positive and PI-negative subpopulation represented cells that were in early apoptosis. Cells that were in late apoptosis or already dead were both Annexin V and PI positive, and necrotic cells were Annexin V negative and PI positive. The apoptotic cell rate was calculated by adding the portion of Annexin V-positive/PI-negative cells and the portion of Annexin V-positive/PI-positive cells.

After treated with CAI and/or sorafenib for 48 hours, cells were harvested and fixed in $75 \%$ ethanol at $4^{\circ} \mathrm{C}$ for 18 hours. After cells were washed twice with cold PBS, DNA staining was performed using PI $(0.05 \mathrm{mg} / \mathrm{ml})$ and RNase $(2 \mathrm{mg} / \mathrm{ml})$ (Beyotime Biotechnology) at room temperature for 30 minutes. Cell cycle distributions were measured by FCM (with an excitation/emission wavelength of $488 \mathrm{~nm} / 617 \mathrm{~nm}$ ). Distributions of cell cycle phases were calculated using ModFit LT software (Verity Software House, Topsham, ME).

Determination of Intracellular ROS Level and Mitochondrial Membrane Potential. Cells were seeded in six-well plates and treated with the indicated drugs. For FCM analysis, cells were detached from the plates with Trypsin-EDTA after 24-hour treatment, washed twice with PBS buffer, and incubated with $20 \mu \mathrm{M} 2^{\prime}, 7^{\prime}$-dichlorofluorescein diacetate (DCFH-DA) (Sigma-Aldrich) for 20 minutes at $37^{\circ} \mathrm{C}$. Cells were then washed gently three times with PBS buffer and were tested using FCM. FCM was performed using an excitation/emission wavelength of $488 / 525 \mathrm{~nm}$ for DCFH-DA.

For fluorescence microscopy detection, cells were washed twice with PBS buffer after 24-hour treatment and incubated with $20 \mu \mathrm{M}$ DCFH-DA for 20 minutes. Cells were then washed gently three times with PBS buffer and were detected using fluorescence microscopy. 
MitoSOX Red is a fluorogenic dye, and higher fluorescence upon oxidation of the probe indicates increased mitochondrial ROS or superoxide (Invitrogen, Carlsbad, CA). Cells were seeded in six-well plates and treated with the indicated drugs. After 24-hour treatment, cells were detached from the plates with Trypsin-EDTA, washed twice with PBS buffer, and incubated with $5 \mu \mathrm{M}$ MitoSOX for 10 minutes at $37^{\circ} \mathrm{C}$. Cells were then washed gently three times with PBS buffer and were tested using FCM. FCM was performed using an excitation/ emission wavelength of $488 / 625 \mathrm{~nm}$ for MitoSOX. In the negative control group, DMSO (solvent of MitoSOX) was used instead of MitoSOX to define the background and delimit the negative regions from the positive regions.

Analysis of Mitochondrial Membrane Potential. To measure the depolarization of mitochondrial membrane potential, JC-1 staining (mitochondrial membrane potential assay kit; Beyotime Biotechnology) was applied. In normal cells (high $\Delta \Psi_{\mathrm{m}}$ ), JC-1 forms as $\mathrm{J}$-aggregates with red fluorescence in the mitochondrial matrix. However, in apoptotic and necrotic cells (low $\Delta \Psi_{\mathrm{m}}$ ), JC-1 remains in monomeric form with green fluorescence. Thus, mitochondrial depolarization is indicated by a decrease in the red/green fluorescence intensity ratio. The cells were seeded in six-well plates and treated with CAI and/or sorafenib. After 24 hours, cells were detached from the plates with Trypsin-EDTA, washed with PBS, resuspended with $500 \mu \mathrm{l} \mathrm{JC}-1$ working solution, and subsequently incubated at $37^{\circ} \mathrm{C}$ for 20 minutes. Finally, cells were washed twice with the assay buffer and FCM analysis was performed. Flow cytometry was performed using excitation/emission wavelengths of $485 / 529 \mathrm{~nm}$ for the monomeric form and 535/590 $\mathrm{nm}$ for the J-aggregates.

LLC Xenograft Model. On day 0, $1 \times 10^{6} \mathrm{LLC}$ cells resuspended in $100 \mu \mathrm{l}$ PBS were injected subcutaneously into the right axillary fossa of 6 -week-old male C57BL/6 mice (Institute of Laboratory Animal Sciences, Chinese Academy of Medical Sciences and School of Basic Medicine, Peking Union Medical College) weighing 18-22 g. Mice were randomly divided into four groups, with 10 animals in each group. The animals were treated by gavage once daily, with either vehicle control (PEG400), CAI (30 mg/kg per day), low-dose sorafenib (SFB-L; $10 \mathrm{mg} / \mathrm{kg}$ per day), high-dose sorafenib (SFB-H; $30 \mathrm{mg} / \mathrm{kg}$ per day), or a combination of CAI (30 mg/kg per day) plus SFB (10 mg/kg per day). Changes in tumor length and width were measured with a caliper every other day from days 10 to 29 , and the tumor volumes (in cubed millimeters) were calculated using the following formula: Volume $=$ length $\times$ width $^{2} \times 0.5$. Mice were euthanized after 29 days of treatment. Tumors were collected and weighed. All animal studies and procedures were approved by the Institutional Animal Care and Use Committee of Peking Union Medical College.

Tumor Sample Preparation. Tumor tissues were homogenized in ice-cold lysis buffer containing T-PER Tissue Protein Extraction Reagent (Thermo Fisher Scientific Inc., Pittsburgh, PA) with Protease and Phosphatase Inhibitor Cocktail (Roche, Mannheim, Germany), incubated for 15 minutes, and then centrifuged at $16,100 \times g$ at $4^{\circ} \mathrm{C}$ for 30 minutes. The protein-containing supernatant was collected and the protein concentration was quantified using the BCA Protein Assay Kit (BioTeke Corporation, Beijing, China).

Western Blot Assay. LLC cell lysates or tumor tissue homogenate containing $30 \mu \mathrm{g}$ proteins was subjected to SDS/PAGE and separated proteins were transferred onto polyvinylidene fluoride membrane. After the membrane was blocked with $5 \%$ nonfat dry milk in Tris-buffered saline containing Tween-20, it was incubated with the desired antibodies. The following primary antibodies were used: NANOG, poly(ADP-ribose) polymerase (PARP), cleaved caspase-3, glyceraldehyde 3-phosphate dehydrogenase (all from Cell Signaling Technology Inc., Danvers, MA), and $\beta$-actin (Sigma-Aldrich). Subsequently, the membrane was incubated with the appropriate secondary antibody and the immunoreactive protein bands were visualized using a chemiluminescence kit (Millipore, Billerica, MA) followed by ECL-based autoradiography (GE Healthcare, Hertfordshire, UK). Western blots are representative of at least three independent experiments.

Immunohistochemistry Staining. Tumor sections from a C57bearing LLC transplant model were baked at $60^{\circ} \mathrm{C}$ for 20 minutes, deparaffinized with xylene, and rehydrated in graded ethanol series. After antigen retrieval and endogenous peroxidase activity blocking, the slides were stained for NANOG (ab80892, dilution 1:1000; Abcam, Cambridge, UK). Localization of specific reactivity was detected using a secondary antibody conjugated to peroxidase followed by observation with 3,3'-diaminobenzidine (DAB) substrate (Zhongshan Golden Bridge Biotechnology, Beijing, China). Slides were counterstained with hematoxylin.

Total RNA Extraction and Reverse Transcription Quantitative Polymerase Chain Reaction. RNA was isolated from LLC cells using the Pure RNA Extraction Kit (BioTeke Corporation) and $2 \mu \mathrm{g}$ total RNA was used to synthesize cDNA with TransScript FirstStrand cDNA Synthesis Supermix (TransGen Biotech Co., Beijing, China), per the manufacturer's instructions. Quantitative polymerase
A
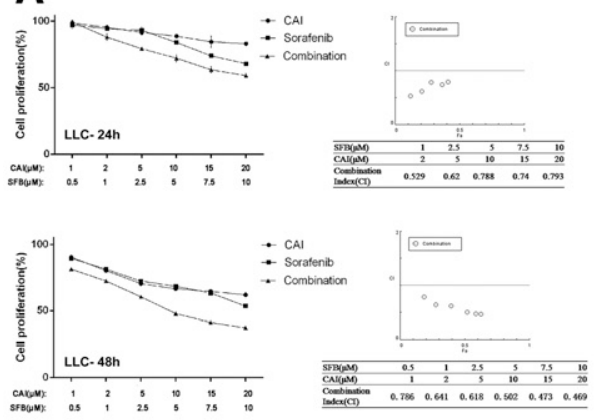

: CAI
: Coratenito
- Combination

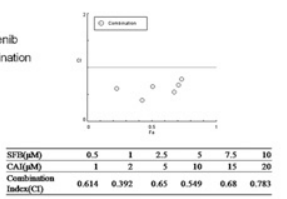

B
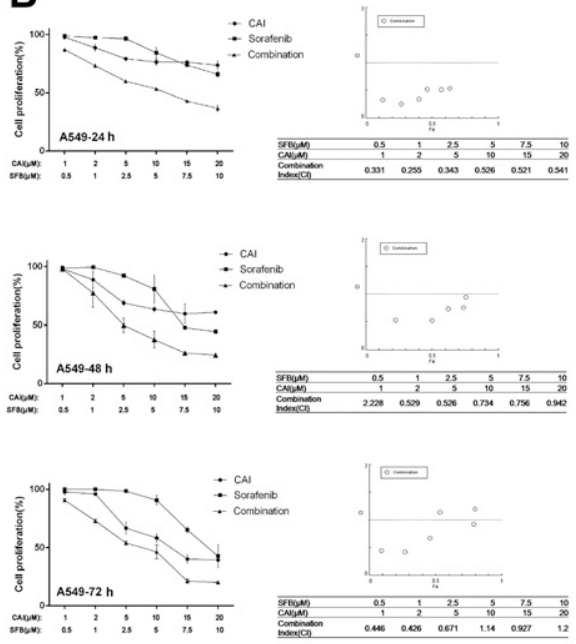

C
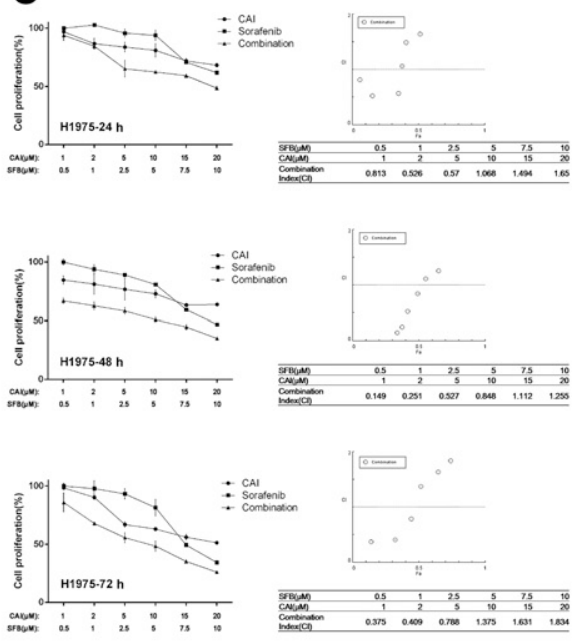

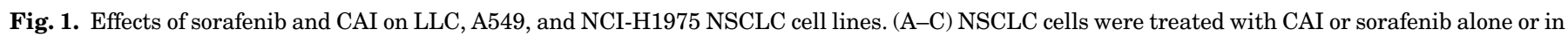

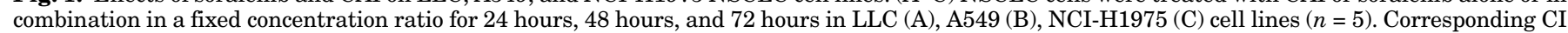
values are shown to the right of each graph. 
chain reaction was performed using the IQ5 Real-Time System (BioRad, Hercules, CA). Each reverse transcription quantitative polymerase chain reaction $(25 \mu \mathrm{l})$ contained $2 \mu \mathrm{l}$ diluted cDNA and $12.5 \mu 12 \times$ Maxima SYBR Green (Thermo Fisher Scientific Inc.). All reactions were performed using the following thermal cycling conditions: $94^{\circ} \mathrm{C}$ for 2 minutes followed by 40 cycles of a two-step reaction, denaturation at $94^{\circ} \mathrm{C}$ for 10 seconds, and annealing at $60^{\circ} \mathrm{C}$ for 30 seconds followed by a melting curve from 55 to $95^{\circ} \mathrm{C}$ in 1 -second increments of $0.5^{\circ} \mathrm{C}$ to ensure amplification specificity. Transcript levels of the target genes were normalized to glyceraldehyde 3-phosphate dehydrogenase.

Malondialdehyde Assay. Tumor tissues were homogenized and prepared for malondialdehyde (MDA) assay according to the manufacturer's instructions in the Lipid Peroxidation MDA assay kit (Beyotime Biotechnology). The MDA concentration of each sample was detected at $532 \mathrm{~nm}(450 \mathrm{~nm}$ as a control) by a microplate reader (Synergy 4; BioTeke Corporation).
Statistical Analysis. All data are expressed as means \pm S.D. of three independent experiments unless otherwise indicated. All data were analyzed by the $t$ test or analysis of variance followed by the Tukey multiple-comparisons test. $P$ values $<0.05$ were considered statistically significant.

\section{Results}

Effects of Sorafenib and CAI on LLC, A549, and H1975 NSCLC Cell Lines. To measure the response of NSCLC cells to sorafenib and/or CAI, we first performed cell proliferation assays. We incubated the LLC, A549, and H1975 cell lines with the respective drugs and their combinations. Sorafenib at concentrations of $0.5,1,2.5,5,7.5$, and $10 \mu \mathrm{M}$ and CAI at concentrations of $1,2,5,10,15$, and $20 \mu \mathrm{M}$ and their combination were all applied. As shown in Fig. 1, CAI,

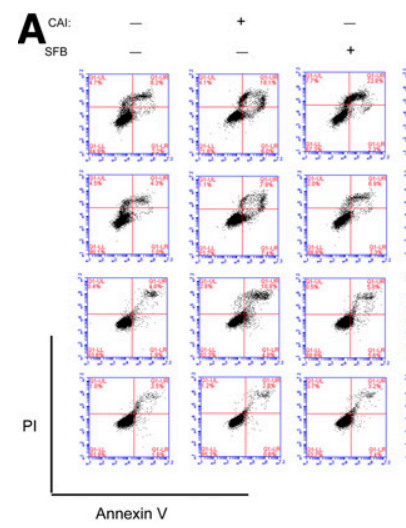

B
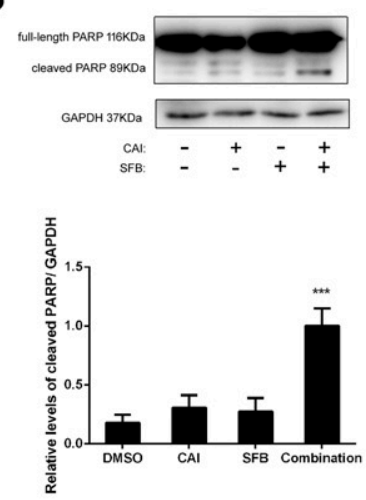

D

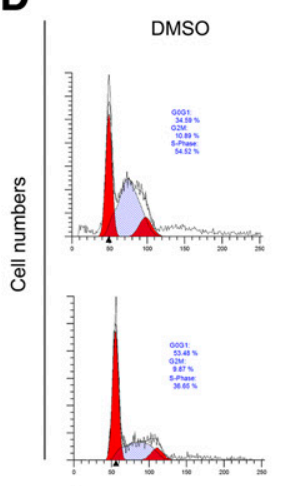

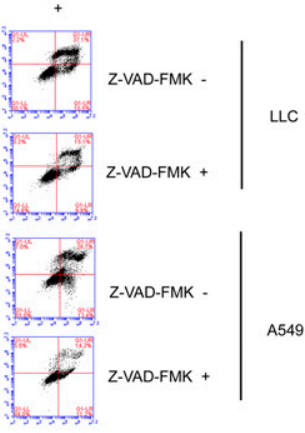
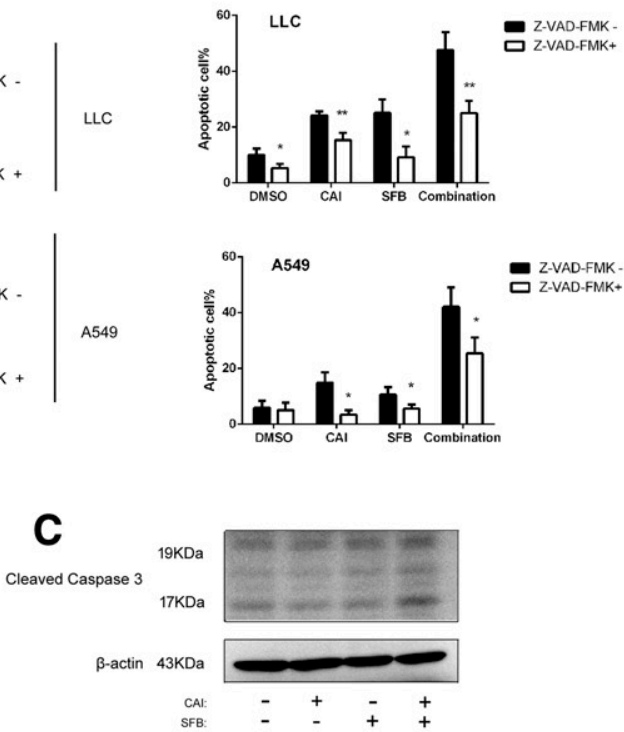

I

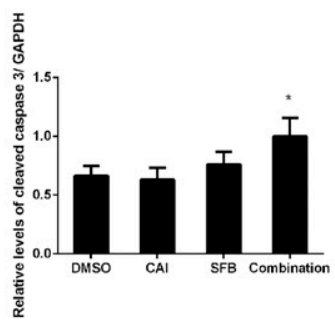

SFB

Combination
Fig. 2. The combination of sorafenib and CAI induces apoptosis in NSCLC. (A) LLC cells were treated with $10 \mu \mathrm{M}$ CAI and/or $5 \mu \mathrm{M}$ sorafenib, and the DMSO group was treated with or without $20 \mu \mathrm{M}$ Z-VAD-FMK for 48 hours. The cells were then stained with Annexin V/PI and analyzed for apoptosis by flow cytometry. Statistical quantifications of apoptotic cells are shown in the graphs on the right. Values are presented as means \pm S.D. $* P<0.05 ; * * P<0.01$ (significant between groups cotreated with or without Z-VADFMK). (B and C) Effect of $10 \mu \mathrm{M}$ CAI and/or $5 \mu \mathrm{M}$ sorafenib on the expression of cleaved PARP (B) and cleaved caspase-3 (C). Protein levels of cleaved PARP and cleaved caspase-3 from treated cell lysates were normalized against GAPDH levels. Band density was evaluated using ImageJ software (National Institutes of Health, Bethesda, MD). Values are presented as means \pm S.D. $* P<0.05$, *** $P<0.001$ (significant compared with DMSO) (D) Cell cycle analysis was conducted in LLC and A549 cells using flow cytometry. Cells were treated with CAI $(10 \mu \mathrm{M})$ and/or sorafenib (5 $\mu \mathrm{M})$ for 24 hours. The results are representative of three independent experiments $(n=5)$. GAPDH, glyceraldehyde 3-phosphate dehydrogenase.
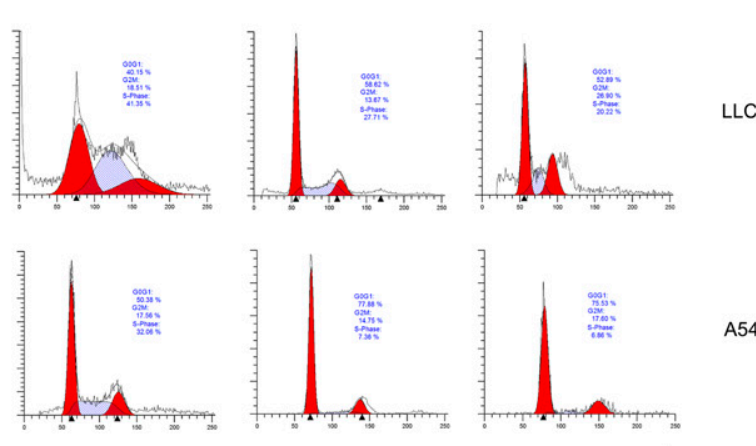

DNA content 
sorafenib, and their combination led to dramatically reduced cell proliferation, in a dose-dependent manner, in all three cell lines investigated. It is noteworthy that the cytotoxic effect of a sorafenib dose as low as $1 \mu \mathrm{M}$ in combination with CAI was comparable to $10-15 \mu \mathrm{M}$ sorafenib alone in all three cell lines and at three time points. The three cell lines were treated with different concentrations of $\mathrm{CAI}$ and sorafenib at a fixed ratio (2: 1), using median effect analysis (Chou and Talalay, 1984). As indicated in Fig. 1, the CI values for all 5 combination groups at 3 indicated time points were less than 1 in LLC cells (Fig. 1A). And in A549 cells CI < 1 was observed for all 5 combination groups when cells were incubated with the combinations for $24 \mathrm{~h}$ and $48 \mathrm{~h}$. The CI values for 3 lower-dose combination groups were less than 1 at $72 \mathrm{~h}$ (Fig. 1B). And similar situation that lowerdose combination showed smaller CI appeared in H1975 cells treated with 5 combinations of CAI and sorafenib for various time (Fig. 1C). This means that the combination of sorafenib and CAI led to synergistic inhibition of cell proliferation in LLC cells, and the combination of both drugs, especially in a lower dose, generated a synergistic effect in the A549 and H1975 cell lines.

The Combination of Sorafenib and CAI Induces Apoptosis in NSCLC. Since the synergistic inhibitory effect of sorafenib and CAI on NSCLC specific mechanisms of cell death must be further explored. To clarify the effects of combining these two agents, LLC cells were treated with the drugs individually or in combination, and Annexin V/propidium iodide staining was used to evaluate apoptosis. After 48 hours, sorafenib $(5 \mu \mathrm{M})-$ treated and CAI (10 $\mu \mathrm{M})$-treated LLC cells elicited $24.1 \%$ and $25.1 \%$ apoptosis, respectively, and the combination induced a larger proportion of cell apoptosis (up to 47.6\%). In A549 cells, CAI-, sorafenib- or the combination-induced apoptotic cell percentage was $14.8 \%, 10.6 \%$, and $42.1 \%$, respectively. In addition, an increased portion of necrotic cells (Annexin $\mathrm{V}$ negative/PI positive) were observed in the combination group (Fig. 2A).

To further confirm the induction of apoptosis by this combination, we analyzed cell extracts for expression of PARP (an endogenous substrate of caspase-3 and caspase-7) and cleaved caspase-3 (which is associated with programmed cell death) (Fig. 2, B and C). The levels of cleaved PARP and cleavedl caspase 3 were elevated in the combination group, indicating that cell was executed by programmed cell death. To verify whether the increase in late apoptotic cells treated with CAI and/or sorafenib was associated with caspase signaling, cells were cotreated with $20 \mu \mathrm{M}$ Z-VAD-FMK, a pan-caspase inhibitor. The apoptotic percentage of cells cotreated with Z-VAD-FMK was significantly decreased compared with treatment with the indicated drugs alone (Fig. 2A). These results show that the combination of CAI and sorafenib synergistically induced apoptosis in vitro. Cell cycle analysis showed that CAI-treated LLC and A549 cells were arrested in the G2/M phase and sorafenib-treated cells were arrested in the G0/G1 phase. In addition, the combination group showed a significant decrease in the $\mathrm{S}$ phase and an increase in both the G0/G1 and G2/M phases (Fig. 2D).

Sorafenib and CAI Increase DCFH-DA and MitoSOX Oxidation Suggesting Increased Intracellular ROS and Provoke Mitochondrial Depolarization. Abnormal accumulation of intracellular ROS could induce oxidative stress in cancer cells, and excessive ROS could directly
A
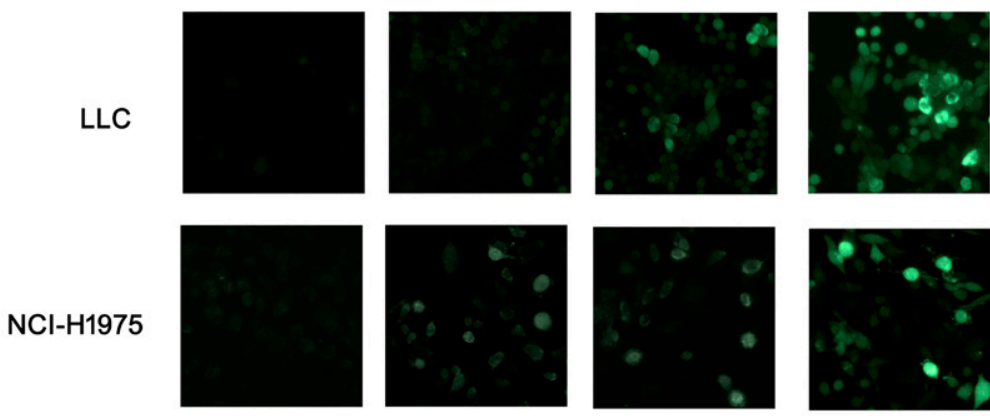

CAI:

SFB:

B

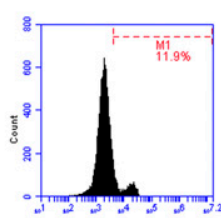

DMSO

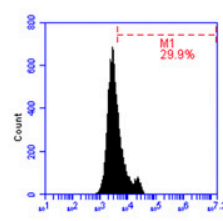

SFB

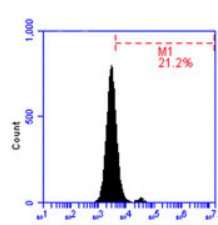

CAI

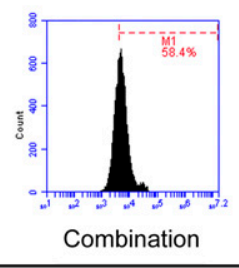

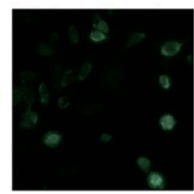

-

$+$

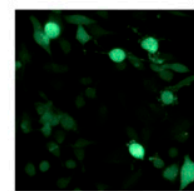

$+$

$+$

C

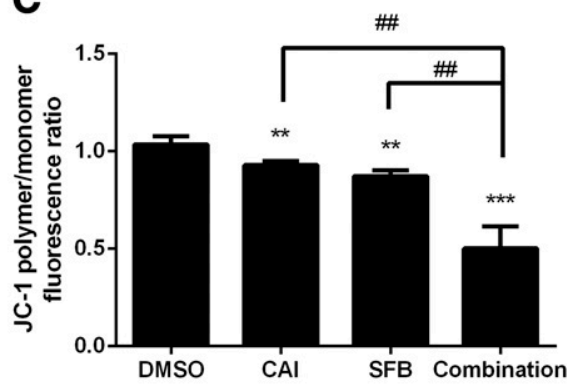

Fig. 3. Sorafenib and CAI increase DCFH-DA and MitoSOX oxidation, suggesting increased intracellular ROS, and provoke mitochondrial depolarization. (A) LLC and H1975 cells were incubated with DMSO $(0.1 \%)$, sorafenib $(5 \mu \mathrm{M})$, and/or CAI $(10 \mu \mathrm{M})$ for 24 hours, and cellular ROS was evaluated by DCFH-DA oxidation. (B) LLC cells were treated with DMSO $(0.1 \%)$, sorafenib $(5 \mu \mathrm{M})$, and/or CAI $(10 \mu \mathrm{M})$ for 24 hours, and MitoSOX was added before flow cytometric analysis. (C) Mitochondrial membrane potential was determined by JC-1 dye and measured by flow cytometric analysis $(n=5)$. $* * P<0.01$; $* * * P<0.001$ (significant compared with DMSO); ${ }^{\# \#} P<0.01$ (significant between monotherapy groups and the combination group).

MitoSOX fluorescence intensity 
attack DNA, protein, lipids, and other cellular components, eventually leading to cell apoptosis (Simon et al., 2000). Previous studies showed that sorafenib or CAI alone could induce the generation of intracellular ROS both in vivo and in vitro (Corrado et al., 2011; Coriat et al., 2012). Therefore, we speculated that the synergistic effect of sorafenib and CAI on apoptosis might enhance the abnormal level of ROS. We first used DCFH-DA as a probe and observed dichlorofluorescein fluorescence under a fluorescence microscope. In LLC and H1975 cell lines, ROS production increased slightly in the presence of sorafenib or CAI, whereas it was provoked significantly in the combination group (Fig. 3A). We further observed enhanced fluorescence intensity upon increased MitoSOX oxidation after CAI, sorafenib, or combination treatment, suggesting that these interventions could increase mitochondrial ROS or superoxide at varying degrees in LLC cells (Fig. 3B).

Since we found that mitochondrial released ROS contributed to the accumulation of the overall ROS in cells. Furthermore, high ROS levels normally disrupted mitochondrial membrane potential $\left(\Delta \Psi_{\mathrm{m}}\right)$. In addition, mitochondrial depolarization is a distinctive feature of the early stages of programmed cell death. Thus, we determined changes in $\Delta \Psi_{\mathrm{m}}$ by staining cells with JC-1 dye. Compared with DMSO group, CAI $(10 \mu \mathrm{M})$ or sorafenib (5 $\mu \mathrm{M})$ in LLC cells as monotherapy slightly decreased $\Delta \Psi_{\mathrm{m}}$, whereas the combination group was dramatically damaged mitochondrial function as decreased $50 \%$ the $\Delta \Psi_{\mathrm{m}}$ compared with DMSO control. Thus, these data revealed that CAI and
A

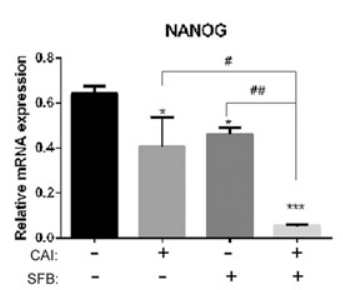

C

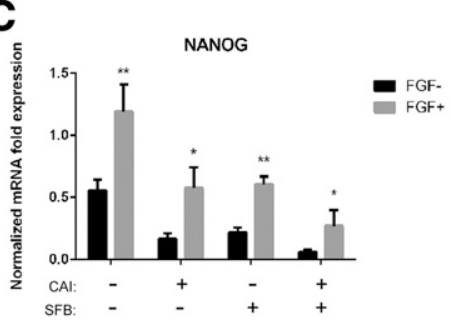

$\mathbf{E}$
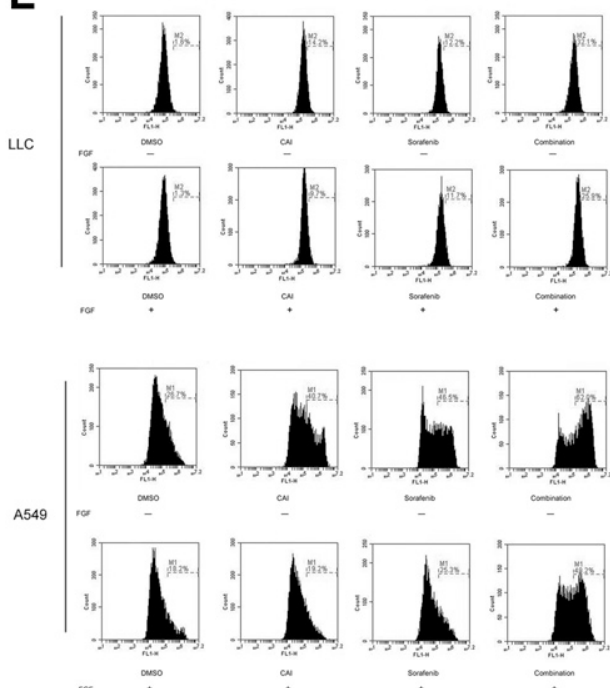

B
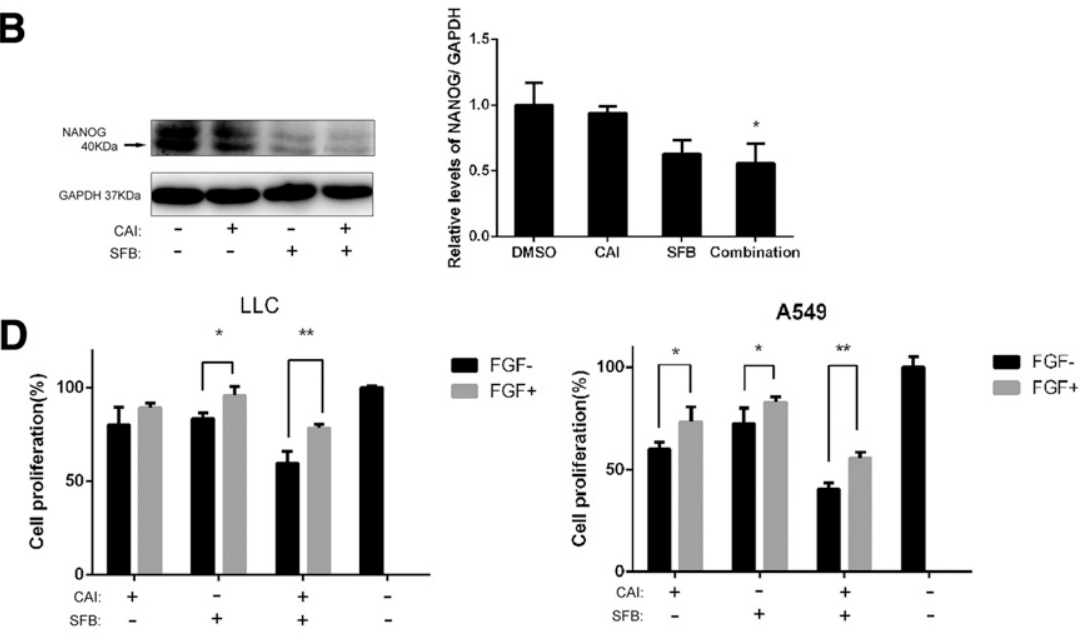

$\mathbf{F}$

LLC

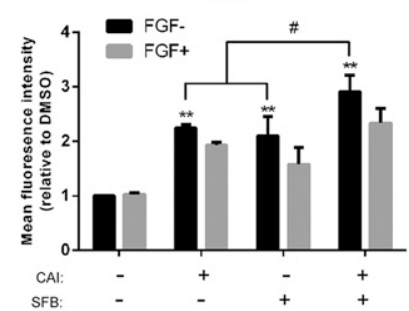

G

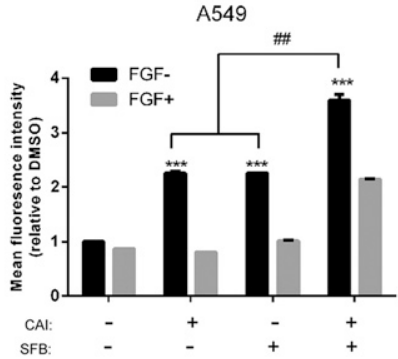

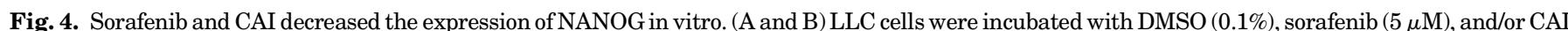

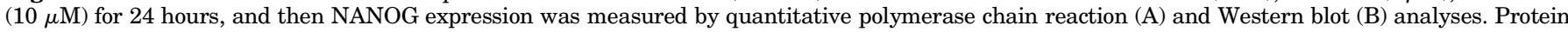

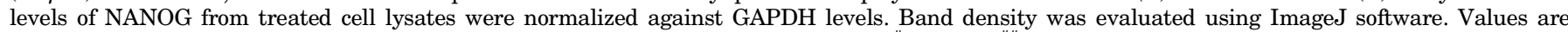

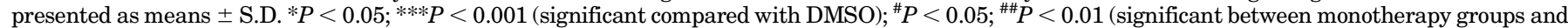

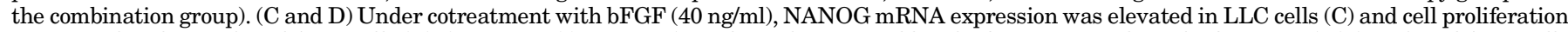

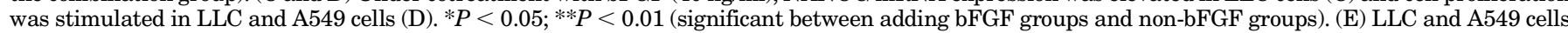

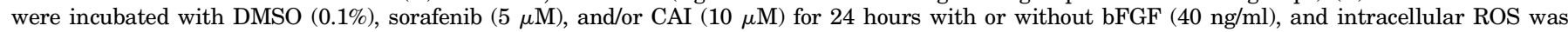

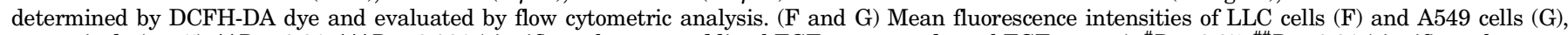

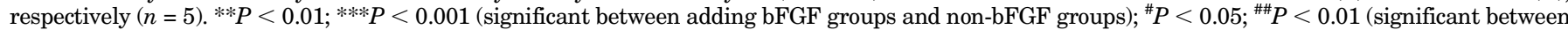
monotherapy groups and the combination group). FGF, fibroblast growth factor; GAPDH, glyceraldehyde 3-phosphate dehydrogenase. 
sorafenib induced ROS accumulation and sabotaged mitochondrial potential.

Sorafenib and CAI Decreased the Expression of NANOG In Vitro. Several studies reveal that NANOG is not only a marker of stem cells but also a key factor in endowing cancer cells with certain stem cell characteristics such as metastasis, tumorigenicity, self-renewal, and drug resistance (Gong et al., 2015). To investigate the role of NANOG in CAI- and/or sorafenib-induced anticancer activity, we measured mRNA and protein levels of NANOG after exposing LLC cells to the indicated treatment. As shown in Fig. 4A, after 24-hour treatment with CAI or sorafenib, NANOG mRNA levels decreased slightly in single-agent groups compared with the control, whereas the combination of CAI and sorafenib was more effective in restraining NANOG expression than the drugs used as monotherapy (Fig. 4A). Similarly, this tendency was confirmed by Western blot experiments (Fig. 4B).

To explore whether NANOG is involved in the accumulation of ROS after CAI and/or sorafenib treatment, we used basic fibroblast growth factor (bFGF), which is reported to stimulate NANOG expression in cancer cells (Shen et al., 2016). In this study, bFGF stimulated NANOG expression in LLC cells and promoted both LLC and A549 cell proliferation (Fig. 4, C and D). In A549 and LLC cell lines, cotreatment with bFGF significantly reduced the CAI- and/or sorafenib-mediated increase in intracellular ROS levels (Fig. 4, E and F). Strikingly, in A549 cells, cotreatment with bFGF almost inhibited the ability in increasing ROS of CAI or sorafenib as monotherapy (Fig. 4G). These results implied that NANOG expression was impaired by CAI in combination with sorafenib and the decrease of its expression was, at least partially, responsible for the accumulation of ROS.

Sorafenib and CAI Induced Apoptosis in a ROS and NANOG-Dependent Manner In Vitro. Sorafenib and CAI instigated aberrant accumulation of ROS, which were associated with the downregulation of NANOG expression. To further verify the role of NANOG and ROS in CAI- and/or sorafenibinduced cell apoptosis, bFGF and GSH were used concomitantly with the indicated drugs. As shown in Fig. 5, A and C, $40 \mathrm{ng} / \mathrm{ml} \mathrm{bFGF}$ and $4 \mathrm{mM}$ GSH partially reverted the effect of sorafenib and/or CAI on cell apoptosis. These data show that ROS production and NANOG inhibition are important upstream pathways of sorafenib- and/or CAI-induced cell apoptosis. In addition, increased amounts of necrotic cells (Annexin V negative/PI positive) were observed in experiments with the sorafenib and CAI combination treatment (Fig. 5, A and C).

The Combination of Sorafenib and CAI Exhibited Synergistic Antitumor Activity In Vivo. The data from our in vitro experiments incited us to evaluate the efficiency of this combination in an in vivo model. Thus, C57BL/6J mice bearing the LLC tumor model were established. Mice were gavaged with vehicle (PEG400), SFB-L ( $10 \mathrm{mg} / \mathrm{kg}$ body weight), CAI (30 mg/kg body weight), SFB-H (30 mg/kg body weight), or combination treatment with sorafenib $(10 \mathrm{mg} / \mathrm{kg}$ body weight) and CAI (30 $\mathrm{mg} / \mathrm{kg}$ body weight) every day for 29 days.

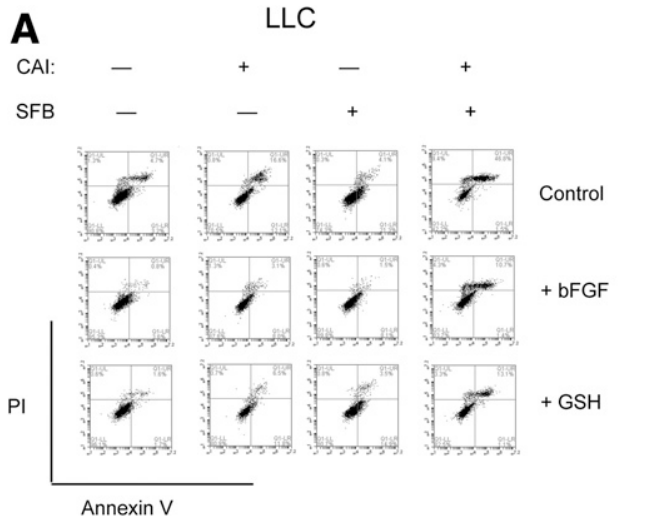

B
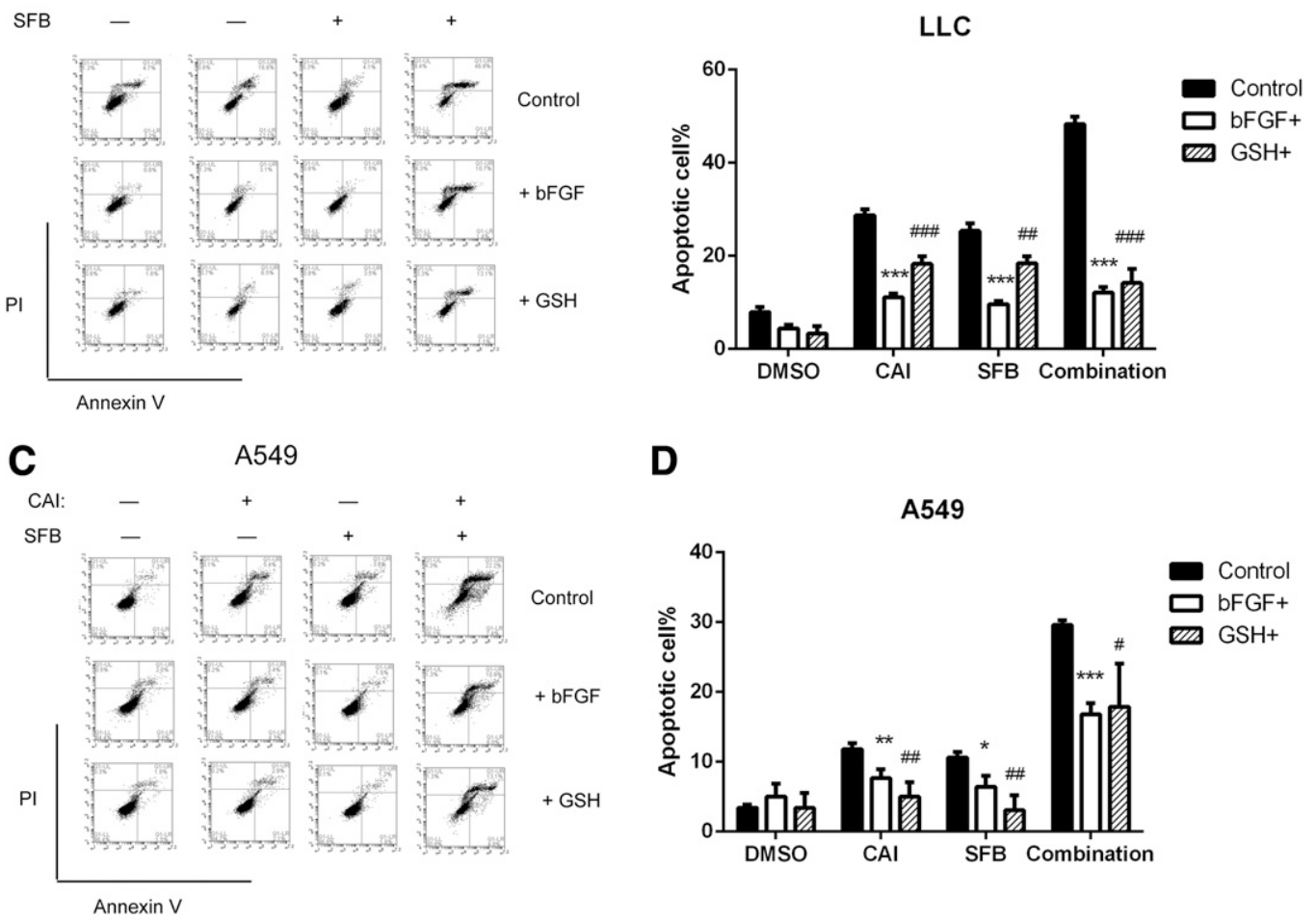

Fig. 5. Sorafenib and CAI induced apoptosis in a ROS- and NANOG-dependent manner in vitro. (A and C) LLC cells (A) and A549 cells (C) were cotreated with $40 \mathrm{ng} / \mathrm{ml} \mathrm{bFGF}$ or $4 \mathrm{mM}$ GSH with sorafenib $(5 \mu \mathrm{M})$ and/or CAI (10 $\mu \mathrm{M})$ for 48 hours, after which cells were stained with Annexin V/ PI and analyzed for apoptosis by flow cytometry. (B and D) Statistical quantification of apoptotic LLC cells (B) and A549 cells (D), respectively. Values are presented as means $\pm \mathrm{S} / \mathrm{D}$. ${ }^{*} P<0.05 ;{ }^{* *} P<0.01 ; * * * P<0.001$ (significant between control groups and in cotreatment with $\mathrm{bFGF}$ groups); ${ }^{*} P<0.05$;

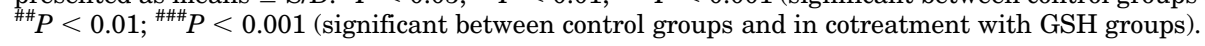


Combination treatment led to less tumor growth compared with single-drug treatment (Fig. 6A). We then assessed the tumor weight to further verify the efficiency of this combination (Fig. 6B). In accordance with tumor volumes, the average tumor weights associated with PEG400, CAI, SFB-L, SFB-H, and combination treatment were 4.77, 2.56, 2.14, $1.68 \mathrm{~g}$, and 1.59 , respectively. It is worth noting that the dose of sorafenib was $10 \mathrm{mg} / \mathrm{kg}$ in the combination group and $30 \mathrm{mg} / \mathrm{kg}$ in the SFB-H group; there was no statistically significant difference in tumor weight between two groups (Fig. 6B).

To evaluate side effects on mice in general, we monitored their weight daily. The average body weights (arranged from high to low) were $29.58 \mathrm{~g}$ (PEG400), $29.14 \mathrm{~g}$ (CAI), $27.64 \mathrm{~g}$ (combination), $27.18 \mathrm{~g}$ (SFB-L), and $26.66 \mathrm{~g}$ (SFB-H) (Fig. 6D).
A

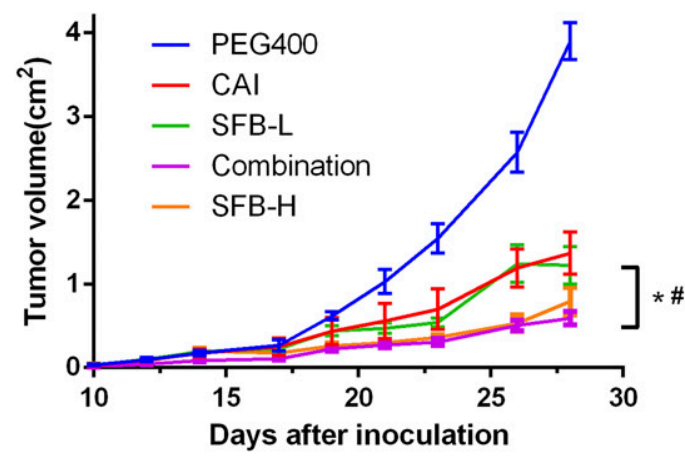

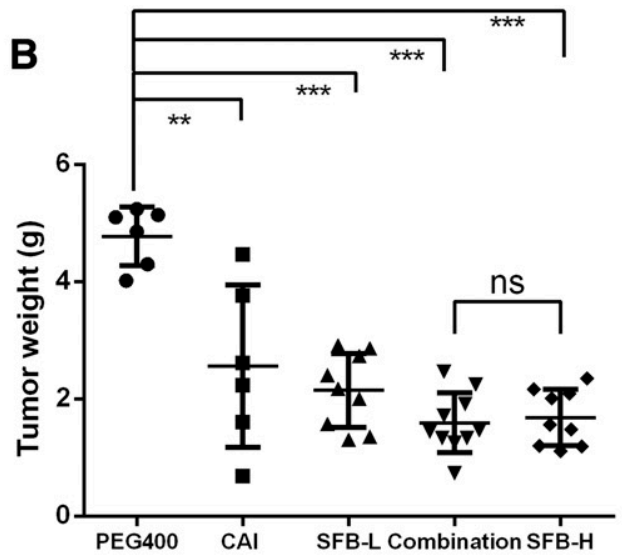

\section{C}

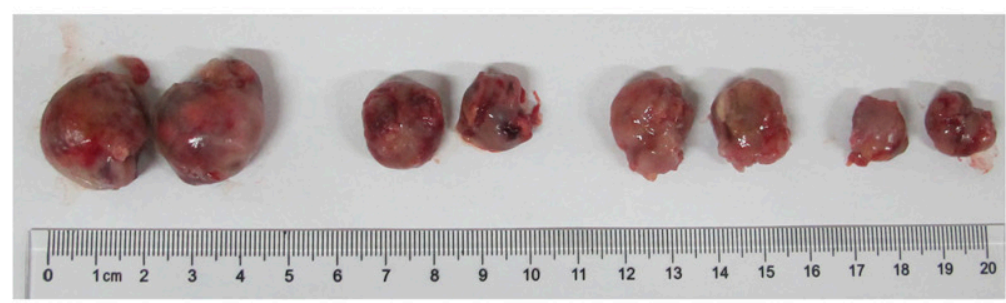

PEG 400

CAl

Sorafenib

Combination
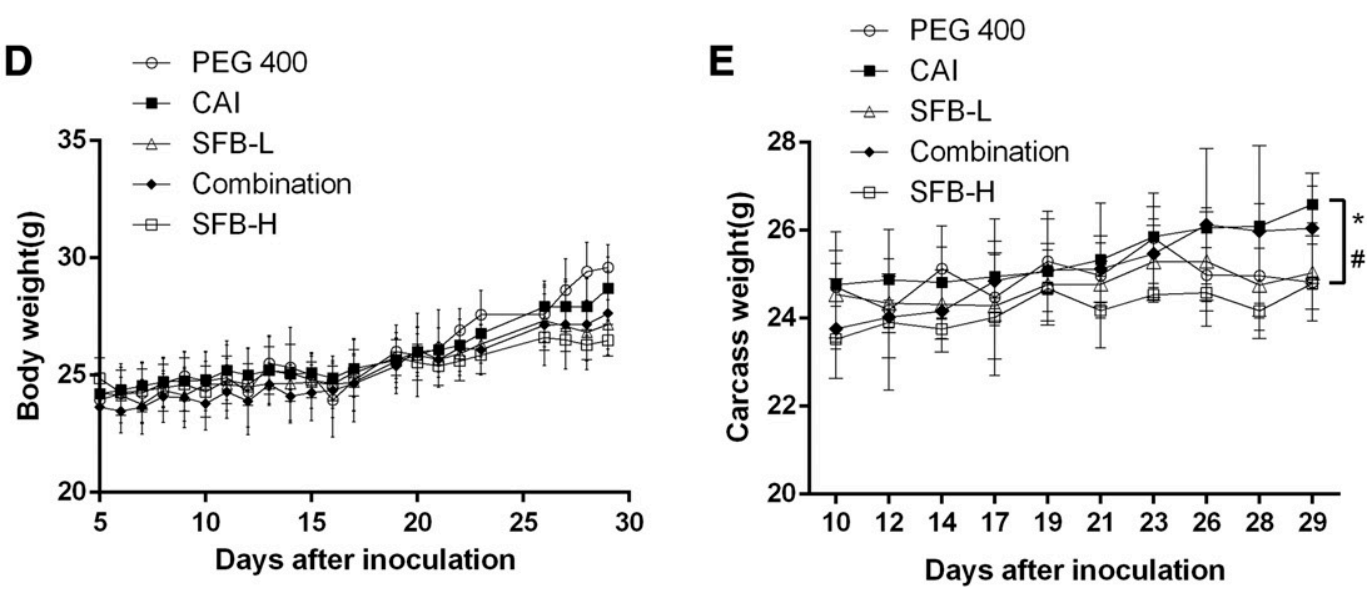

Fig. 6. The combination of sorafenib and CAI exhibits synergistic antitumor activity in vivo. Male C57BL/6J mice bearing LLC tumors were randomly distributed into five groups $(n=10)$ and treated with PEG400, CAI (30 mg/kg), SFB-L (10 mg/kg), SFB-H (30 mg/kg), or the combination of CAI (30 mg/kg) and SFB $(10 \mathrm{mg} / \mathrm{kg})$. (A) Tumor volumes were measured with calipers every other day. Mean tumor volumes \pm S.E.M. of each group are shown $(n=6-10)$. ${ }^{*} P<0.05$ (significant between combination group with the CAI group); ${ }^{\#} P<0.05$ (significant between the combination group with SFB-L group). (B) On day 29, tumors were excised and weighed. As a result of tumor progression, 10 mice died before the end of the experiment (4 in the PEG400 group, 4 in the CAI group, 1 in the SFB-L group, and 1 in the SFB-H group). Tumor weights are presented as scatter dots. ** $P<0.01 ; * * * P<0.001$ (significant compared with the PEG400 group). (C) Photograph of tumors in the PEG400, CAI, SFB-L, and combination groups. (D) Body weights of mice in each group were measured daily to roughly evaluate the side effects of different treatments. Mean body weights \pm S.E.M. of each group are $\operatorname{shown}(n=6-10)$. (E) To exclude the effect of differences in tumor weights in body weight, we calculated carcass weights by using body weight minus the corresponding tumor weight. $* P<0.05$ (significant between CAI group with PEG400 group); ${ }^{\#}<0.05$ (significant between combination group with SFB-H group). ns, not significant. 
Although the PEG400 group seemed to have the highest body weight compared with other groups, the tumor weight in the PEG400 group was much higher than the others (as mentioned earlier). Therefore, we calculated the carcass weight of each group to exclude the difference in tumor weights. As shown in Fig. 6E, the carcass weight exhibited a slightly different tendency: $26.59 \mathrm{~g}$ (CAI), $26.05 \mathrm{~g}$ (combination), $25.04 \mathrm{~g}$ (SFB-L), $24.82 \mathrm{~g}$ (PEG400), and $24.80 \mathrm{~g}$ (SFB-H). Consistent with our previous observation that CAI could ameliorate cancer-associated cachexia (Chen et al., 2017), the CAI and combination treatment groups showed potential to maintain the body weight of tumor-bearing mice compared with the PEG400 group. It is noteworthy that the average carcass weight in the combination group was significantly heavier than that in the SFB-H group (Fig. 6E). Taken together, these data suggest that CAI not only synergized in vivo antitumor activity with sorafenib but also attenuated weight loss during cancer progression.

Sorafenib and CAI Inhibit NANOG In Vivo. Because in vitro studies indicate that NANOG is a key mediator of CAI and sorafenib- induced cell death, we also investigated the expression of NANOG in vivo. We performed immunohistochemistry for NANOG expression in tumor sections after 29 days of treatment with sorafenib and/or CAI (Fig. 7A). The percentage of NANOG-stained tumor sections was significantly decreased in the combination group compared with groups treated with vehicle or single agents. We used Western blot analysis to test tumor lysis in each group, and we further confirmed that NANOG expression was decreased in the combination group (Fig. 7B). In addition, hematoxylin and eosin staining revealed that greater necrosis was observed in tumors after treatment with sorafenib and CAI compared with vehicle or single agents (Fig. 7A). The quantification of MDA is commonly applied as a marker for lipid peroxidation and is often seen at the level of ROSmediated injury. The MDA level was increased in the combination group compared with the other groups (Fig. 7C). Taken together, these data confirmed that NANOG was downregulated after CAI and sorafenib treatment in vivo.

\section{Discussion}

We report here the synergistic antitumor effect of sorafenib and CAI in NSCLC both in vivo and in vitro. The underlying mechanisms of this effect are associated with downregulation of NANOG and induction of apoptosis. Combination
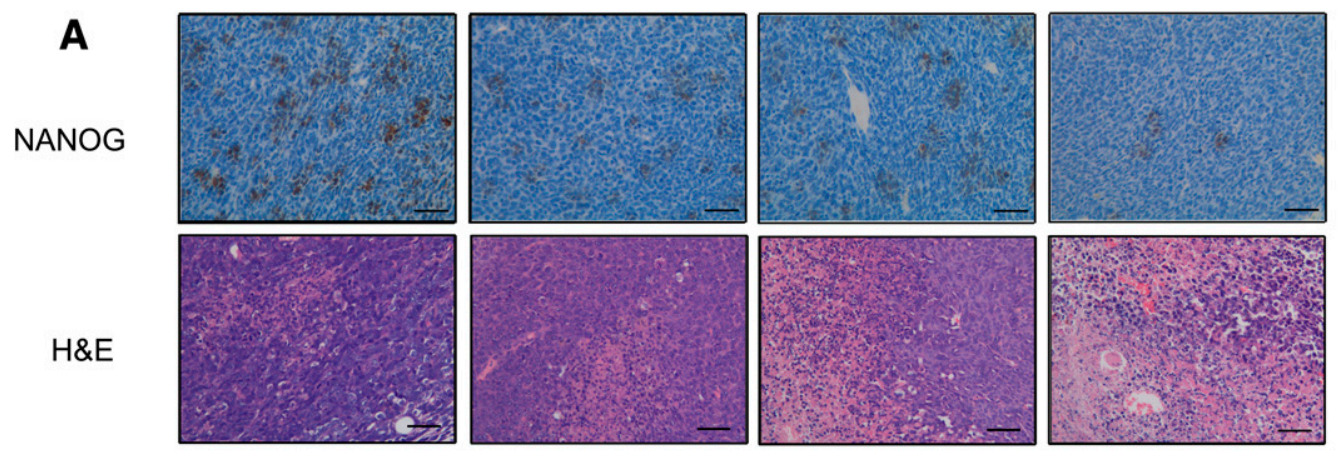

PEG400

CAI

SFB-L
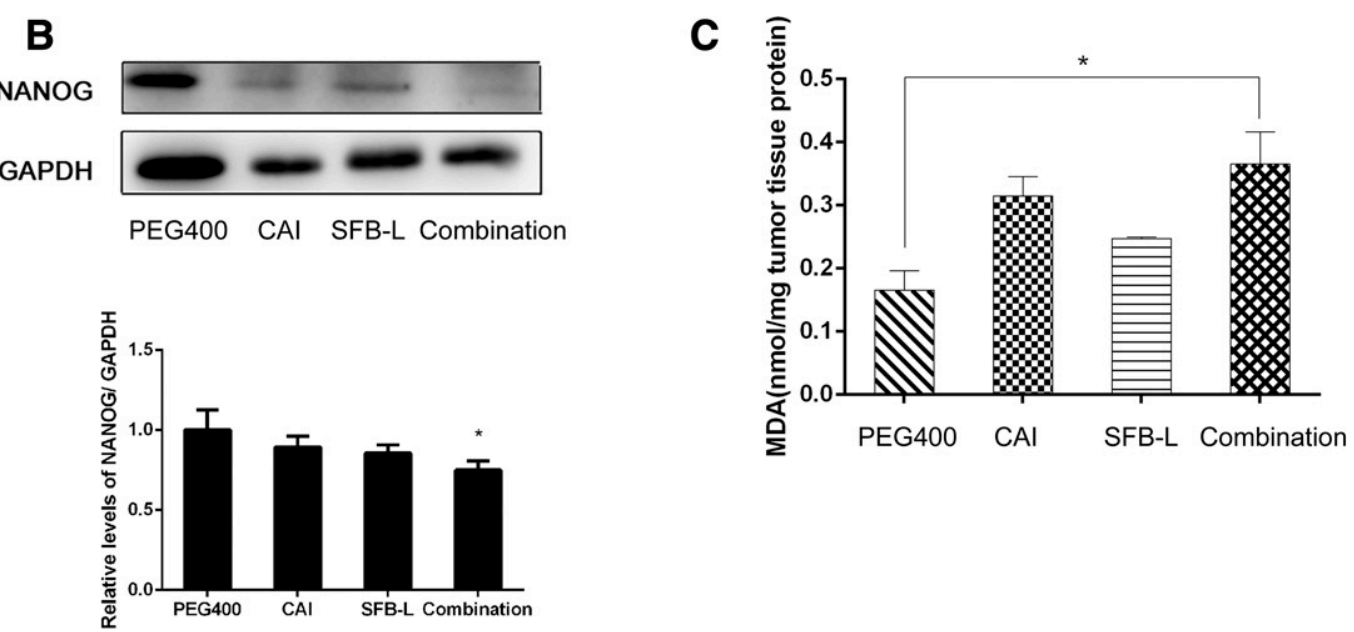

Fig. 7. Sorafenib and CAI inhibit NANOG in vivo. (A) Immunohistochemistry of NANOG and H\&E staining were performed on tumor sections in the PEG400, CAI, SFB-L, and combination groups. (B) Tumor lysis was prepared using tumors excised from mice at the end of the experiment. Levels of NANOG in tumor lysates of PEG400, CAI, SFB-L, and combination groups were measured using Western blot analysis. Protein levels of NANOG from tumor lysates were normalized against GAPDH levels. Band density was evaluated using ImageJ software. Values are presented as means \pm SD. $* P<$ 0.05 (versus PEG400 group). (C) Tumor lysates were subjected to the MDA assay. $* P<0.05$. GAPDH, glyceraldehyde 3 -phosphate dehydrogenase; H\&E, hematoxylin and eosin. 
therapy-induced apoptosis was mostly associated with mitochondrial-related cell death, owing to the accumulation of mitochondrial ROS, upregulation of cleaved caspase-3, and depolarization of mitochondria.

NANOG has an important role in tumorigenesis, chemoresistance, relapse, and metastasis. A previous study showed that NANOG-positive CSCs were much more resistant to sorafenib than NANOG-negative CSCs (Shan et al., 2012). Here, we found that the combination of CAI and sorafenib could dramatically decrease mRNA and protein levels of NANOG, which to some extent transferred NANOG-positive cells to a state of NANOG-negative cells. This might be a reason why, NSCLC cells were more sensitive to sorafenib in combination with CAI both in vivo and in vitro. To better understand the exact mechanism by which the combination treatment dramatically inhibits NANOG, the upstream signaling of NANOG perturbed by these two agents must be further explored.

It was recently shown that in TICs, NANOG combines with the promoter of OXPHOS genes and restrains their expression, quenching ROS production (Chen et al., 2016). OXPHOS defects are a well known pivotal reason for the reduction in apoptosis in cancer cells (Yadav et al., 2015). The downregulation of NANOG may result in restored expression of OXPHOS-encoding genes. Our previous study showed that CAI, like rotenone, could inhibit the enzyme activity of one OXPHOS components (complex I) (Ju et al., 2016). It is clear that rotenone can block the electron transport chain, resulting in OXPHOS blockade and ROS accumulation (Radad et al., 2006). Similarly, it is possible that CAI may induce ROS production in a similar manner; thus, OXPHOS gene expression may necessitate exposing complex I to CAI and may trigger downstream signaling.

It is well known that the excessive generation of ROS by various chemotherapies leads to the loss of mitochondrial membrane potential and induces apoptosis in cancer cells. In addition, ROS are also key factors for chemoresistance of cancer. In LLC and A549 cells, using bFGF to upregulate NANOG is associated with decreased levels of ROS, even in CAI, sorafenib or combination groups. At the same time, with decreased ROS production, cell proliferation was also promoted by the cotreatment of bFGF. This implies that ROS accumulation by CAI and/or sorafenib occurs partially through downregulation of NANOG. In addition, the augment of ROS by these two agents is possibly associated with OXPHOS expressions.

Interestingly, CI values of A549 cells were mostly less than 1, which means that the combination of CAI and sorafenib at different concentrations was synergistic. However, in H1975 cells, CI values were less than 1 in a lower dosage of both drugs and more than 1 in higher dosages, which indicated that the combination lost efficacy in the high dosages. It is well known that A549 cells possess wild-type endothelial growth factor receptor (EGFR) and KRAS mutations (exon 2), whereas H1975 cell harbors the L858R/T790M EGFR mutations and wild-type KRAS. The absence of the synergistic effect of CAI and sorafenib in H1975 cells might be due to the mutation of EGFR, since $L 858 R / T 790 M$ double mutants display a considerable increase in tyrosine phosphorylation levels compared with only one mutation in either L858R or T790M (Mulloy et al., 2007). The combination regimen may not be able to counteract the aberrant activation of EGFR signaling. Mutations in the KRAS protooncogene are common aberrations and are found in $10 \%-30 \%$ of lung adenocarcinomas. These mutations lead to abnormal activation of the KRAS signaling pathway that results in continuous cell proliferation and indicates poor NSCLC prognosis. The combination of CAI and sorafenib has a synergistic effect in cells regardless of KRAS mutations. These data imply that patients with KRAS mutations and without L858R / T790M EGFR mutations will benefit from the synergistic effect of CAI and sorafenib.

In conclusion, combined CAI and sorafenib therapy has a synergistic effect in resisting NSCLC in vivo and in vitro, which may contribute to the induction of apoptosis and inhibition of NANOG. The combination in vivo may allow a dose reduction of sorafenib and may avoid decreasing effectiveness. Thus, our findings provide a novel strategy to expand the application of sorafenib and may raise a new choice for treatment of NSCLC.

\section{Authorship Contributions}

Participated in research design: C. Chen, Zhang, Ye, Guo.

Conducted experiments: C. Chen, Ju, Shi, W. Chen.

Contributed new reagents or analytic tools: $\mathrm{Zhu}, \mathrm{Li}$.

Performed data analysis: C. Chen, Sun.

Wrote or contributed to the writing of the manuscript: C. Chen, Guo, and Ye.

\section{References}

Alessandro R, Fontana S, Giordano M, Corrado C, Colomba P, Flugy AM, Santoro A, Kohn EC, and De Leo G (2008) Effects of carboxyamidotriazole on in vitro models of imatinib-resistant chronic myeloid leukemia. J Cell Physiol 215:111-121.

Batchelor TT, Sorensen AG, di Tomaso E, Zhang WT, Duda DG, Cohen KS, Kozak KR, Cahill DP, Chen PJ, Zhu M, et al. (2007) AZD2171, a pan-VEGF receptor tyrosine kinase inhibitor, normalizes tumor vasculature and alleviates edema in glioblastoma patients. Cancer Cell 11:83-95.

Berlin J, Tutsch KD, Hutson P, Cleary J, Rago RP, Arzoomanian RZ, Alberti D, Feierabend C, and Wilding G (1997) Phase I clinical and pharmacokinetic study of oral carboxyamidotriazole, a signal transduction inhibitor. J Clin Oncol 15: 781-789.

Blumenschein GR, Jr, Gatzemeier U, Fossella F, Stewart DJ, Cupit L, Cihon F, O'Leary J, and Reck M (2009) Phase II, multicenter, uncontrolled trial of singleagent sorafenib in patients with relapsed or refractory, advanced non-small-cell lung cancer. J Clin Oncol 27:4274-4280.

Chen C, Ju R, Zhu L, Li J, Chen W, Zhang DC, Ye CY, and Guo L (2017) Carboxyamidotriazole alleviates muscle atrophy in tumor-bearing mice by inhibiting NF-кB and activating SIRT1. Naunyn Schmiedebergs Arch Pharmacol 390: 423-433.

Chen CL, Uthaya Kumar DB, Punj V, Xu J, Sher L, Tahara SM, Hess S, and Machida K (2016) NANOG metabolically reprograms tumor-initiating stem-like cells through tumorigenic changes in oxidative phosphorylation and fatty acid metabolism. Cell Metab 23:206-219.

Chou TC (2006) Theoretical basis, experimental design, and computerized simulation of synergism and antagonism in drug combination studies. Pharmacol Rev 58: $621-681$.

Chou TC and Talalay P (1984) Quantitative analysis of dose-effect relationships: the combined effects of multiple drugs or enzyme inhibitors. Adv Enzyme Regul 22:27-55. Clark JW, Eder JP, Ryan D, Lathia C, and Lenz HJ (2005) Safety and pharmacokinetics of the dual action Raf kinase and vascular endothelial growth factor receptor inhibitor, BAY 43-9006, in patients with advanced, refractory solid tumors. Clin Cancer Res 11:5472-5480.

Coriat R, Nicco C, Chéreau C, Mir O, Alexandre J, Ropert S, Weill B, Chaussade S, Goldwasser F, and Batteux F (2012) Sorafenib-induced hepatocellular carcinoma cell death depends on reactive oxygen species production in vitro and in vivo. Mol Cancer Ther 11:2284-2293.

Corrado C, Raimondo S, Flugy AM, Fontana S, Santoro A, Stassi G, Marfia A, Iovino F, Arlinghaus R, Kohn EC, et al. (2011) Carboxyamidotriazole inhibits cell growth of imatinib-resistant chronic myeloid leukaemia cells including T315I Bcr-Abl mutant by a redox-mediated mechanism. Cancer Lett 300:205-214.

Desai AA, Innocenti F, Janisch L, DeMario M, Shepard D, Ramirez J, Fleming $\mathrm{GF}$, and Ratain MJ (2004) A phase I trial of pharmacokinetic modulation of carboxyamidotriazole (CAI) with ketoconazole in patients with advanced cancer. Cancer Chemother Pharmacol 54:377-384.

Du Y, Ma C, Wang Z, Liu Z, Liu H, and Wang T (2013) Nanog, a novel prognostic marker for lung cancer. Surg Oncol 22:224-229.

Dutcher JP, Leon L, Manola J, Friedland DM, Roth B, and Wilding G; Eastern Cooperative Oncology Group (2005) Phase II study of carboxyamidotriazole in patients with advanced renal cell carcinoma refractory to immunotherapy: E4896, an Eastern Cooperative Oncology Group Study. Cancer 104:2392-2399.

Gong S, Li Q, Jeter CR, Fan Q, Tang DG, and Liu B (2015) Regulation of NANOG in cancer cells. Mol Carcinog 54:679-687.

Gridelli C, Maione P, Del Gaizo F, Colantuoni G, Guerriero C, Ferrara C, Nicolella D, Comunale D, De Vita A, and Rossi A (2007) Sorafenib and sunitinib in the treatment of advanced non-small cell lung cancer. Oncologist 12:191-200. 
Hirsch FR, Scagliotti GV, Mulshine JL, Kwon R, Curran WJ, Jr, Wu YL, and PazAres L (2017) Lung cancer: current therapies and new targeted treatments. Lancet 389:299-311.

Hussain MM, Kotz H, Minasian L, Premkumar A, Sarosy G, Reed E, Zhai S, Steinberg SM, Raggio M, Oliver VK, et al. (2003) Phase II trial of carboxyamidotriazole in patients with relapsed epithelial ovarian cancer. J Clin Oncol 21:4356-4363.

Jemal A, Bray F, Center MM, Ferlay J, Ward E, and Forman D (2011) Global cance statistics. CA Cancer J Clin 61:69-90.

Johnson EA, Marks RS, Mandrekar SJ, Hillman SL, Hauge MD, Bauman MD, Wos EJ, Moore DF, Kugler JW, Windschitl HE, et al. (2008) Phase III randomized, double-blind study of maintenance CAI or placebo in patients with advanced nonsmall cell lung cancer (NSCLC) after completion of initial therapy (NCCTG 97-24 51). Lung Cancer 60:200-207.

Ju R, Guo L, Li J, Zhu L, Yu X, Chen C, Chen W, Ye C, and Zhang D (2016) Carboxyamidotriazole inhibits oxidative phosphorylation in cancer cells and exerts synergistic anti-cancer effect with glycolysis inhibition. Cancer Lett 370:232-241.

Kohn EC, Figg WD, Sarosy GA, Bauer KS, Davis PA, Soltis MJ, Thompkins A, Liotta LA, and Reed E (1997) Phase I trial of micronized formulation carboxyamidotriazole in patients with refractory solid tumors: pharmacokinetics, clinical outcome, and comparison of formulations. J Clin Oncol 15:1985-1993.

Langer CJ, Mok T, and Postmus PE (2013) Targeted agents in the third-/fourth-line treatment of patients with advanced (stage III/IV) non-small cell lung cancer (NSCLC). Cancer Treat Rev 39:252-260.

Lin YL, Han ZB, Xiong FY, Tian LY, Wu XJ, Xue SW, Zhou YR, Deng JX, and Chen HX (2011) Malignant transformation of 293 cells induced by ectopic expression of human Nanog. Mol Cell Biochem 351:109-116.

Mikkelsen T, Lush R, Grossman SA, Carson KA, Fisher JD, Alavi JB, and Rosenfeld S (2007) Phase II clinical and pharmacologic study of radiation therapy and carboxyamido-triazole (CAI) in adults with newly diagnosed glioblastoma multiforme. Invest New Drugs 25:259-263.

Moore M, Hirte HW, Siu L, Oza A, Hotte SJ, Petrenciuc O, Cihon F, Lathia C, and Schwartz B (2005) Phase I study to determine the safety and pharmacokinetics of the novel Raf kinase and VEGFR inhibitor BAY 43-9006, administered for 28 days on/7 days off in patients with advanced, refractory solid tumors. Ann Oncol 16:1688-1694.

Mulloy R, Ferrand A, Kim Y, Sordella R, Bell DW, Haber DA, Anderson KS, and Settleman J (2007) Epidermal growth factor receptor mutants from human lung cancers exhibit enhanced catalytic activity and increased sensitivity to gefitinib. Cancer Res 67:2325-2330.

Paz-Ares L, Hirsh V, Zhang L, de Marinis F, Yang JC, Wakelee HA, Seto T, Wu YL, Novello S, Juhász E, et al. (2015) Monotherapy administration of sorafenib in patients with non-small cell lung cancer (MISSION) trial: a phase III, multicenter, placebo-controlled trial of sorafenib in patients with relapsed or refractory predominantly nonsquamous non-small-cell lung cancer after 2 or 3 previous treatment regimens. J Thorac Oncol 10:1745-1753.
Radad K, Rausch WD, and Gille G (2006) Rotenone induces cell death in primary dopaminergic culture by increasing ROS production and inhibiting mitochondrial respiration. Neurochem Int 49:379-386.

Shan J, Shen J, Liu L, Xia F, Xu C, Duan G, Xu Y, Ma Q, Yang Z, Zhang Q, et al. (2012) Nanog regulates self-renewal of cancer stem cells through the insulin-like growth factor pathway in human hepatocellular carcinoma. Hepatology 56: 1004-1014.

Shen C, Ka SO, Kim SJ, Kim JH, Park BH, and Park JH (2016) Metformin and AICAR regulate NANOG expression via the JNK pathway in HepG2 cells independently of AMPK. Tumour Biol 37:11199-11208.

Simon HU, Haj-Yehia A, and Levi-Schaffer F (2000) Role of reactive oxygen species (ROS) in apoptosis induction. Apoptosis 5:415-418.

Strumberg D, Clark JW, Awada A, Moore MJ, Richly H, Hendlisz A, Hirte HW, Eder JP, Lenz HJ, and Schwartz B (2007) Safety, pharmacokinetics, and preliminary antitumor activity of sorafenib: a review of four phase I trials in patients with advanced refractory solid tumors. Oncologist 12:426-437.

Vichai V and Kirtikara K (2006) Sulforhodamine B colorimetric assay for cytotoxicity screening. Nat Protoc 1:1112-1116.

Wakelee HA, Lee JW, Hanna NH, Traynor AM, Carbone DP, and Schiller JH (2012) A double-blind randomized discontinuation phase-II study of sorafenib (BAY 43-9006) in previously treated non-small-cell lung cancer patients: eastern cooperative oncology group study E2501. J Thorac Oncol 7:1574-1582.

Wilhelm S, Carter C, Lynch M, Lowinger T, Dumas J, Smith RA, Schwartz B, Simantov R, and Kelley S (2006) Discovery and development of sorafenib: a multikinase inhibitor for treating cancer. Nat Rev Drug Discov 5:835-844.

Wilhelm SM, Carter C, Tang L, Wilkie D, McNabola A, Rong H, Chen C, Zhang X, Vincent P, McHugh M, et al. (2004) BAY 43-9006 exhibits broad spectrum oral antitumor activity and targets the RAF/MEK/ERK pathway and receptor tyrosine kinases involved in tumor progression and angiogenesis. Cancer Res 64 7099-7109.

Yadav N, Kumar S, Marlowe T, Chaudhary AK, Kumar R, Wang J, O’Malley J, Boland PM, Jayanthi S, Kumar TK, et al. (2015) Oxidative phosphorylationdependent regulation of cancer cell apoptosis in response to anticancer agents. Cell Death Dis 6:e1969.

Address correspondence to: Dr. Caiying Ye, Department of Pharmacology, Institute of Basic Medical Sciences, Chinese Academy of Medical Sciences and School of Basic Medicine, Peking Union Medical College, 5, Dongdan Santiao, Beijing 100005, China. E-mail: caiyingye@126.com or Dr. Lei Guo, Department of Pharmacology, Institute of Basic Medical Sciences, Chinese Academy of Medical Sciences and School of Basic Medicine, Peking Union Medical College, 5, Dongdan Santiao, Beijing 100005, China. E-mail: guoleistu@126.com 